\title{
Stage 0 Small Intestinal Adenocarcinoma AJCC v8
}

National Cancer Institute

\section{Source}

National Cancer Institute. Stage O Small Intestinal Adenocarcinoma A/CC v8. NCI Thesaurus. Code C133894.

Stage 0 includes: T is, N0, M0. Tis: High grade dysplasia/carcinoma in situ. N0: No regional lymph node metastasis. M0: No distant metastasis. (AJCC 8th ed.) 